\title{
Comparison of real-time PCR assay with a commercial rapid test based on the immunochromatographic principle for the detection of Chlamydia trachomatis in seminal fluid and urine specimens
}

\author{
Angela Serafini ${ }^{1}$, Cinzia Mei ${ }^{1}$, Roberta Mattei ${ }^{1}$, Mauro De Santi ${ }^{2}$, Giorgio Brandi ${ }^{2}$ \\ ${ }^{1}$ Biolab - Laboratori Analisi Cliniche Convenzionati, Istituto di Ricerca, Pesaro, Italy; \\ ${ }^{2}$ Dipartimento di Scienze Biomolecolari, Unità di Igiene, Università di Urbino Carlo Bo, Urbino, Italy
}

Article received 20 July, 2020; accepted 24 October, 2021

\section{SUMMARY}

Chlamydia trachomatis is the most common sexually transmitted bacterial infection worldwide, with the highest prevalence in sexually active young women. Since most chlamydial infections are asymptomatic, facilitating the transmission of the pathogen in the population, regular screening is recommended for at-risk sexually active individuals. Hence, a sensitive, specific, inexpensive diagnostic test that can be executed efficiently and quickly to yield results would be helpful. In this study, we evaluated the performance (sensitivity and specificity) of the Chlamydia Rapid Test Device, an inexpensive rapid test based on immunochromatographic analysis. We then compared these results to those obtained using real-time PCR, the gold standard for C. trachomatis testing. Seminal fluid and urine specimens of male subjects were tested.
The rapid test was applied to 220 seminal fluid samples, showing a sensitivity of $0 \%$. On the other hand, used in 114 urine specimens, the rapid test showed $37.5 \%$ and $100 \%$ sensitivity and specificity, respectively. Hence, the Chlamydia Rapid Test Device was shown to be insufficiently sensitive to be recommended as a screening test and diagnostic method for the detection of $C$. trachomatis in symptomatic and asymptomatic subjects. Finally, the present investigation showed an infection prevalence of $5 \%$ calculated using seminal fluid, with a mean age of 28 years.

Keywords: Chlamydia trachomatis, real-time PCR, immunochromatographic, urine, seminal fluid.

\section{INTRODUCTION}

hlamydia trachomatis is one of the most common causative agents of sexually transmitted infections (STI) worldwide that can lead to pelvic inflammatory disease, ectopic pregnancy, and infertility [1-5]. Different genotypes of $C$. trachomatis

Corresponding author

Giorgio Brandi

E-mail: giorgio.brandi@uniurb.it exist: genotypes A, B, and C, are associated with trachoma; genotypes D-K with conjunctivitis and genito-anal infections; genotypes L1-L3 with lymphogranuloma venereum, an emerging disease in Europe and other high-income countries, especially among human immunodeficiency virus (HIV)infected men who have sex with men. Moreover, infection by L genotypes may result in more invasive disease compared to other genotypes since L1-L3 strains have tropism not only for the epithelial surfaces but also for the lymphatic tissues $[6,7]$. 
The World Health Organization (WHO) reports that over 100 million new cases of $C$. trachomatis occur annually worldwide, most frequently in geographical areas characterized by poor hygienic conditions. In particular, the Western Pacific Region and the Americas recorded the highest incidence of chlamydial infections in both men and women (respectively 39 and 30 million new cases per year) $[8,9]$. C. trachomatis infections represent the most common notifiable sexually transmitted infection both in the US, which reported about $1,600,000$ cases in 2017, and in Europe, where about 409,000 cases were reported in 2017 across 26 EU/EEA member countries, with the United Kingdom accounting for $56 \%$ of those cases [10]. Regarding C. trachomatis infections and gender, it was reported that in women, $70-80 \%$ of genital tract infections with $C$. trachomatis are asymptomatic; however, $15-40 \%$ ascend to the upper genital tract, which can lead to severe sequelae, including pelvic inflammatory disease, infertility and ectopic pregnancy [11]. In men, approximately $50 \%$ of chlamydial infections are asymptomatic and are also a potential source of infection in women [12]. In addition, these asymptomatic infections can cause urinary tract inflammation, sperm DNA damage, and acute epididymitis. Since the infection can persist for up to four years in couples, it could affect their fertility. Overall, in both sexes, most reported cases of $C$. trachomatis involve young people between the ages of 15 and 24 , particularly those who engage in risky sexual behaviors, men who have sex with men, and individuals who tend to change partners frequently or have multiple partners [13, 14].

Since most chlamydial infections are asymptomatic, regular screening is recommended for at-risk sexually active individuals $[15,16]$. Such a screening system would require a diagnostic test that is sensitive, specific, and inexpensive, which can be executed easily and yield results rapidly.

The purpose of this study was to evaluate whether a commercially available rapid test based on the immunochromatographic principle (Chlamydia Rapid Test Device) could represent a useful diagnostic tool for the identification of $C$. trachomatis. The rapid test was applied to different biological samples collected in a diagnostic laboratory from male subjects that required the specific analyses, and the results were then compared to those obtained with the nucleic acid amplification test, considered the gold standard for C. trachomatis diagnosis.

\section{SUBJECTS AND METHODS}

\section{Study population}

Between April 2018 and January 2019, 220 male subjects, ranging from 15 to 67 , who were tested at the Biolab Clinical Analysis Laboratory, Pesaro (Central Italy), provided samples after signing a consent form approved by the laboratory director. In detail, 37 were between 15 and 24 years old $(16.81 \%)$, and 5 of them tested positive for $C$. trachomatis infection, 56 were between 25 and 34 years old $(25.45 \%)$, and 4 of them were positive for the infection, while 70 were between 35 and 44 years old $(31.81 \%)$ and within this age group no subjects positive for infection were found; finally, 57 individuals were over 45 years old $(25.90 \%)$, and two men only tested positive for the infection (Figure 1). The status (symptomatic or asymptomatic) of the subjects was not requested for privacy.

\section{Specimen collection}

Self-collected seminal fluid samples were obtained from each of the participants. After fluidification, the samples were divided into two parts: half was used for immunochromatographic analysis (rapid test) and the other half was used for biomolecular analysis (real-time PCR). Samples for the rapid test were stored at $2-8^{\circ} \mathrm{C}$ and analyzed within $72 \mathrm{~h}$ of the collection, whereas samples for real-time PCR analysis were stored in a freezer at a temperature between $-15^{\circ} \mathrm{C}$ and $-30^{\circ} \mathrm{C}$ until processing. Before beginning the immunochromatographic and biomolecular analyses, the samples were stored in a thermostat at room temperature $\left(15^{\circ} \mathrm{C}-25^{\circ} \mathrm{C}\right)$.

Self-collected first-pass urine $(20 \mathrm{ml})$ specimens obtained from the subjects were stored at $2-8^{\circ} \mathrm{C}$ and analyzed within $24 \mathrm{~h}$ of the collection.

\section{Real-time PCR assay}

Genomic DNA was extracted from $0.5 \mathrm{ml}$ of seminal fluid and $20 \mathrm{ml}$ of urine and purified using the QIAsymphony DSP Virus/Pathogen Midi system (Qiagen), which performs a fully automated purification of nucleic acids using magnetic particle technology that consists of four phases: lysis, bonding, washing and elution.

The Rotor-Gene Q Mdx 5plex HRM subsequently 
amplified the extracted DNA (Qiagen) performed using the artus CT/NG QS-RGQ kit (Qiagen). The kit allows the amplification of two specific targets, a $66 \mathrm{pb}$ region of the $C$. trachomatis genome and an $86 \mathrm{pb}$ region of the cryptic plasmid.

\section{Immunochromatographic assay}

The Chlamydia Rapid Test Device (Innovacon) is a rapid chromatographic immunoassay that uses antibodies specific to the LPS antigen of chlamydia in biological samples (cervical and urethral swabs, urine). In order to extract antigens from chlamydia cells, urine samples $(20 \mathrm{~mL})$, following the addition of distilled water, were centrifuged at 3,000 rpm for 15 minutes. HCL $0.2 \mathrm{~N}$ (solution A) was then added to the urine pellet, mixing to homogenize the suspension. Finally, $0.2 \mathrm{M} \mathrm{NaOH}$ (solution B) was added and shaken by vortexing. During testing, the extracted antigen solution reacts with a chlamydia antibody that is coated onto particles. The mixture migrates up to react with the second chlamydia antibody on the membrane, generating a colored line in the test line region (positive result).

The semen sample, which had not been centrifuged, was put in contact first with Reagent $A$ and then with Reagent B using a sterile swab, and the extracted solution was dispensed into the membrane to allow the generation of a colored line, as reported for urine samples.

\section{RESULTS}

In this study, 220 male subjects, ranging in age from 15 to 67, voluntarily undergoing an analysis of seminal fluid to evaluate the presence of a genital infection, were sampled after signing a consent form. The median age of the participants was 36.5 years (range: $15-67$ years).

In the first set of experiments (Sampling A), 106 seminal fluid samples were assessed for the presence of $C$. trachomatis, comparing the performance characteristics of real-time PCR and the rapid test. A total of three subjects (2.83\%) tested positive for the infection using PCR, while none of the subjects tested positive using the rapid test (Table 1). Given the disappointing sensitivity results (0\%) of the rapid test in seminal fluid, both the seminal fluid and urine of an additional 114 subjects were sampled (Sampling B). Considering all 220 seminal fluid samples (106 from sampling A, 114 from sam- pling B), the results showed that $11(9.64 \%)$ and 0 $(0 \%)$ of the subjects tested positive for $C$. trachomatis using the PCR and rapid test, respectively (Table 1). The rapid test applied to the 220 seminal fluid samples showed a sensitivity of $0 \%$ (IC 0.00-28.4). Of the 114 specimens from sampling B, 3 (2.63\%) tested positive using the rapid assay evaluated in urine samples and $8(7 \%)$ using the PCR evaluated in seminal fluid samples (Table 1); hence, the sensitivity of the rapid test applied to urine samples was $37.5 \%$ (IC, 8.52-75.5), whereas its specificity was $100 \%$ (IC, 96.5-100.0).

Having adopted the real-time PCR as the reference method for the diagnosis of $C$. trachomatis infection, a positive PCR result for the sample was considered as positive for infection. Thus, the prevalence of $C$. trachomatis in seminal fluid samples was $5 \%(n=11 / 220,95 \%$ CI $4.4-11.6)$.

The median age of the infected subjects was 28 years. The highest prevalence (13.5\%) was found in the 15-24 age group, followed by the 25-34 age group (7.14 \% prevalence) (Figure 1$)$.

Table 1 - Clinical performance of Chlamydia Rapid Test Device (rapid test) versus real-time PCR.

\begin{tabular}{|c|c|c|c|c|}
\hline \multicolumn{5}{|c|}{ Seminal fluid (sampling A) } \\
\hline & & \multicolumn{3}{|c|}{ Real-time PCR } \\
\hline & & Positive & Negative & $\begin{array}{c}\text { Total } \\
\text { subjects }\end{array}$ \\
\hline \multirow{3}{*}{$\begin{array}{l}\text { Rapid } \\
\text { test }\end{array}$} & Positive & 0 & 0 & 0 \\
\hline & Negative & 3 & 103 & 106 \\
\hline & Total subjects & 3 & 103 & 106 \\
\hline \multicolumn{5}{|c|}{ Seminal fluid (sampling $A+B$ ) } \\
\hline & & \multicolumn{3}{|c|}{ Real-time PCR } \\
\hline & & Positive & Negative & $\begin{array}{c}\text { Total } \\
\text { subjects }\end{array}$ \\
\hline \multirow{3}{*}{$\begin{array}{l}\text { Rapid } \\
\text { test }\end{array}$} & Positive & 0 & 0 & 0 \\
\hline & Negative & 11 & 209 & 220 \\
\hline & Total subjects & 11 & 209 & 220 \\
\hline \multicolumn{5}{|c|}{ Urine (sampling $A+B$ ) } \\
\hline & & \multicolumn{3}{|c|}{ Real-time PCR } \\
\hline & & Positive & Negative & $\begin{array}{c}\text { Total } \\
\text { subjects }\end{array}$ \\
\hline \multirow{3}{*}{$\begin{array}{l}\text { Rapid } \\
\text { test }\end{array}$} & Positive & 3 & 0 & 3 \\
\hline & Negative & 5 & 106 & 111 \\
\hline & Total subjects & 8 & 106 & 114 \\
\hline
\end{tabular}




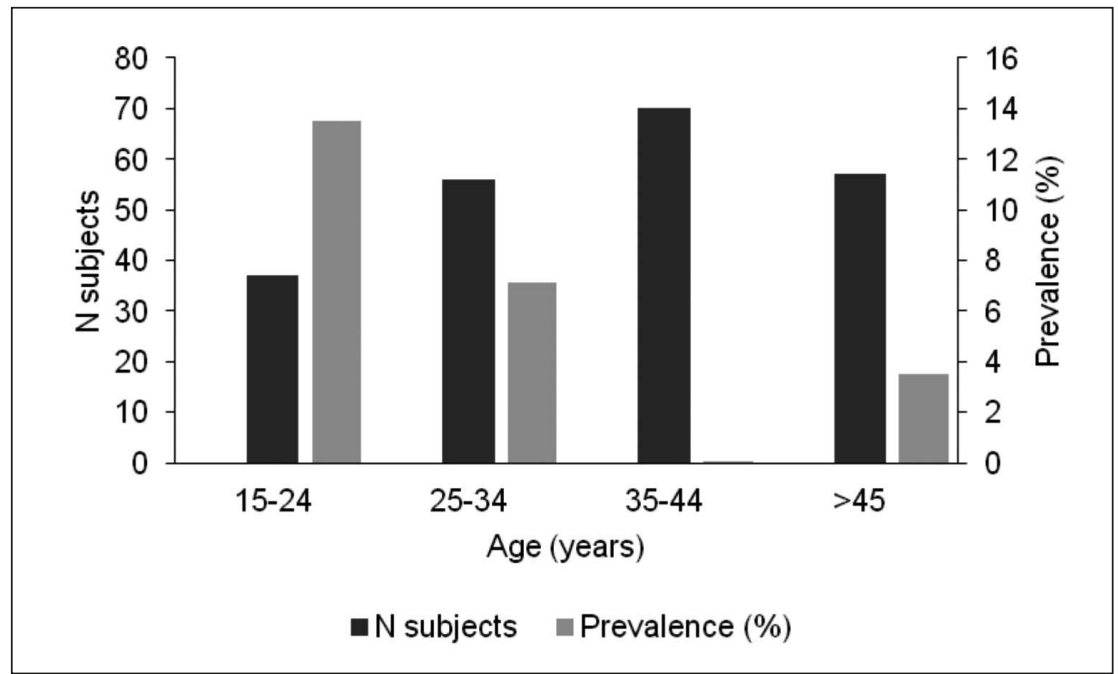

Figure 1 - Prevalence of the infection sustained by Chlamydia trachomatis by age group. The values relating to the number of tested subjects are shown on the left, while the prevalence values, expressed as a percentage, are shown on the right.

Of the 11 subjects infected with $C$. trachomatis, 4 $(36 \%)$ were coinfected with Ureaplasma urealiticum and $2(18 \%)$ with Candida albicans, as shown by sperm culture (not shown).

\section{DISCUSSION}

C. trachomatis infection is asymptomatic in most cases in both sexes and, if untreated, often leads to complications. It would therefore be useful to implement an adequate screening system for both women and men that offers a diagnostic test free of charge to those considered at risk, with the aim of reducing the spread of infection. This kind of screening program requires a sensitive, specific, inexpensive diagnostic test that can be executed easily and yield results quickly.

Currently, numerous laboratory techniques are used for the diagnosis of $C$. trachomatis, starting from different types of biological samples such as urethral swabs, cervical swabs, seminal fluid, and urine (Table 2). Rapid diagnostic tests have recently been developed for the detection of $C$. trachomatis antigens [17-19]. The antigenic specificities characteristic of chlamydiae are represented by MOMP (the main protein of the outer membrane), by LPS (a genus specific lipopolysaccharide), by membrane proteins rich in cysteine (CRP), by thermo shock proteins (HSP) and by a membrane lipoprotein called MIP that stimulates the production of antibodies with neutralizing activity. Rapid immunochromatographic tests generally detect the bacterial LPS antigen [20,21]. The main advantage of these tests consists in their extreme rapidity of execution and, consequently, their ability to yield a qualitative result in about $30 \mathrm{~min}$. The present study was therefore undertaken to evaluate the performance characteristic (sensitivity and specificity) of a rapid, inexpensive test based on immunochromatographic analysis, comparing its results to those obtained using real-time PCR, the gold standard in C. trachomatis testing. Furthermore, this study aims to evaluate whether the rapid test can be validated for the detection of $C$. trachomatis in the seminal fluid used for sperm culture, thus avoiding the collection of urine specimens.

Our results clearly show that the rapid test based on immunochromatographic analysis is not suitable for detecting of C. trachomatis in semen since none of the samples tested positive (sensitivity $0 \%$ ), whereas 11 samples were found to be positive using real-time PCR as a reference method. Thus, seminal fluid cannot be considered a valid, reliable alternative to the matrices provided by the kit (urine and urethral swabs). The discordant results between the rapid test and PCR assay may stem from the fact that the small volume of seminal fluid used in the test $(0.5 \mathrm{ml})$ was insufficient for the detection of antigens, while it may have been sufficient for a method that involves nucleic acid amplification and thus allows a precise diagnosis even in those samples, such as seminal fluid, in which the pathogen may have little or 
Table 2 - Diagnostic tests for $C$. trachomatis.

\begin{tabular}{|c|c|c|}
\hline Diagnosis & Description & Pros/cons \\
\hline Cultural method & $\begin{array}{l}\text { Inclusion bodies identification } \\
\text { (48-72 hours) }\end{array}$ & $\begin{array}{l}\uparrow \text { High sensitivity and selectivity } \\
\downarrow \text { High variability }\end{array}$ \\
\hline IFA & $\begin{array}{l}\text { Immunofluorescence test by the } \\
\text { use of a fluorescein-conjugated } \\
\text { monoclonal antibody }\end{array}$ & $\begin{array}{l}\uparrow \text { Rapid diagnosis (45 min) } \\
\downarrow \text { Low sensitivity }\end{array}$ \\
\hline EIA/ELISA & $\begin{array}{l}\text { Immunoenzymatic tests for } \\
\text { antigens (e.g. LPS, MOMP, ...) }\end{array}$ & $\begin{array}{l}\uparrow \text { Automatization } \\
\downarrow \text { Low sensitivity } \\
\end{array}$ \\
\hline PCR-based tests (NAAT) & $\begin{array}{l}\text { Amplification of C. trachomatis } \\
\text { DNA }\end{array}$ & $\begin{array}{l}\uparrow \text { High sensitivity and precise diagnosis with null/low } \\
\text { amount of viable bacteria } \\
\downarrow \text { High cost and specialized personnel required }\end{array}$ \\
\hline $\begin{array}{l}\text { Immunochromatographic } \\
\text { tests }\end{array}$ & Detection of specific antigens & $\begin{array}{l}\uparrow \text { Rapid tests and low costs } \\
\downarrow \text { Low sensitivity }\end{array}$ \\
\hline $\begin{array}{l}\text { Microimmunofluorescence } \\
\text { Tests (MIFT) }\end{array}$ & Detection of specific antibodies & $\uparrow$ Useful for chronic, invasive infection or re-infection \\
\hline Cytologic analysis & $\begin{array}{l}\text { Colorimetric detection } \\
\text { of inclusion bodies } \\
\text { (May- Grunwald Giemsa) }\end{array}$ & $\begin{array}{l}\uparrow \text { Useful for ocular infection } \\
\downarrow \text { Low sensitivity (not recommended for genital } \\
\text { or conjunctival infections }\end{array}$ \\
\hline
\end{tabular}

zero viability or a very low microbial charge [22]. This finding agrees with the Centers for Disease Control and Prevention (CDC) and the Sexually Transmitted Infections (STI) diagnostic laboratory recommendations that state that infection with $C$. trachomatis should be diagnosed using susceptible and specific nucleic acid amplification tests [3]. Furthermore, real-time PCR also allows the quantification of the amplification product $[17,18]$. Our assessment of the sensitivity of the rapid test applied to urine samples showed a diagnostic performance $(37.5 \%)$ that was well below that reported by the manufacturers (91\%). The difference in sensitivity between the rapid test performed on different samples, $0 \%$ and $37.5 \%$ in semen and urine, respectively, is probably due to differences in the bacterial loads in these samples. However, in any case, in the present study, the Chlamydia Rapid Test Device showed insufficient sensitivity to be recommended as a screening test and diagnostic tool to detect $C$. trachomatis in symptomatic and asymptomatic subjects. Furthermore, this rapid test is not useful for determining the outcome of treatments because antigens may persist following antibiotic therapy.

Although real-time PCR is currently the gold standard for the diagnosis of $C$. trachomatis, nucleic acid amplification techniques, however, have some disadvantages, including their relatively high cost and the fact that they must usually be performed in central laboratories, which involves the transport of samples and a consequent delay in the communication of results (about 3-5 days). Hence, given the high frequency of asymptomatic subjects, it would be useful to develop rapid screening tests with greater specificity and sensitivity to manage the spread of the infection in the population. Such tests would be beneficial in areas where access to nucleic acid amplification tests may be limited.

Finally, the prevalence of infection in the sample group assessed in the present study was $5 \%$ when calculated based on seminal fluid testing, with a higher prevalence $(13.5 \%)$ in the $15-24$ age group, and $7 \%$ when calculated based on urine sample testing. In both cases, however, prevalence is in agreement with the Italian national data, and since the aim of this study was to evaluate the performance of diagnostic kits, the prevalence data are weakly indicative. The findings in this study are subject to some limitations. First, the small volume of seminal fluid obtained from patients compromises antigen detection, resulting in low sensitivity of diagnosis. Secondly, for privacy reasons, the health status of the subject is unknown, making impossible the correlation between C. trachomatis and symptoms.

In conclusion, real-time PCR is currently the gold standard for the diagnosis of C. trachomatis, and the work also suggests that systematic screening 
that is still focused exclusively on women should also be extended to men considered at risk to help further reduce the spread of infection.

\section{Conflicts of interest}

The authors declare that they have no conflict of interest.

\section{Funding \\ None.}

\section{REFERENCES}

[1] Newman L, Rowley J, Vander Hoorn S, et al. Global estimates of the prevalence and incidence of four curable sexually transmitted infections in 2012 based on systematic review and global reporting. PLoS One 2015; 10:e0143304. https://doi.org/10.1371/journal. pone. 0143304 .

[2] World Health Organization (WHO). Sexually transmitted infections (STIs). http://www.who.int/en/ news-room/factsheets/detail/sexually-transmittedinfections-(stis) 2017.

[3] Papp JR, Schachter J, Gaydos CA, Van Der Pol B. Recommendations for laboratory-based detection of Chlamydia trachomatis and Neisseria gonorrhoeae. MMWR Morb Mortal Wkly Recom \& Rep. 2014; 63, 1-19.

[4] Morré SA, Moes R, Van Valkengoed I, et al. Genotyping of Chlamydia trachomatis in urine specimens will facilitate large epidemiological studies. J Clin Microbiol 1998; 36, 3077-8.

[5] Millman K, Black CM, Johnson RE, et al. Populationbased genetic and evolutionary analysis of Chlamydia trachomatis urogenital strain variation in the United States. J Bacteriol. 2004; 186, 2457-65. doi: 10.1128/ jb.186.8.2457-2465.2004.

[6] de Vrieze NH, de Vries HJ. Lymphogranuloma venereum among men who have sex with men. An epidemiological and clinical review. Expert Rev Anti Infect Ther. 2014; 12: 697-704.

[7] Ciccarese G, Drago F, Rebora A, Parodi A. Updates on lymphogranuloma venereum. J Eur Acad Dermatol Venereol. 2021; 35 (8), 1606-7. doi: 10.1111/jdv.17468.

[8] World Health Organisation (WHO) (2017). Global strategy for the prevention and control of sexually transmitted infections: 2006-2015: breaking the chain of transmission. ttp://apps.who.int/iris/bitstream/ handle10665/43853/9789241563475_eng.pdf;jsessioni d=B5D9ED847DD81C1795D67FFECE7D98AF?sequen ce $=1$. Accessed 08 June 2017.

[9] Rowley J, Vander Hoorn S, Korenromp E, et al. Chlamydia, gonorrhoea, trichomoniasis and syphilis: global prevalence and incidence estimates (2016). Bull World Health Organ. 2019; 97 (8), 548-62. doi: 10.2471/ BLT.18.228486.
[10] European Centre for Disease Prevention and Control (ECDC) (2019). Chlamydia infection. In: ECDC. Annual epidemiological report for 2017. Stockholm: ECDC; https://www.ecdc.europa.eu/en/publicationsdata/chlamydia-infection-annual-epidemiological-report-2017.

[11] Malhotra M, Sood S, Mukherjee A, et al. Genital Chlamydia trachomatis: an update. Indian J Med Res. 2013; 138 (3), 303-16.

[12] Peipert JF. Clinical practice. Genital chlamydial infections. N Engl J Med 2003; 349, 2424-30. doi: 10.1056/ NEJMcp030542.

[13] Jonsson M, Karlsson R, Persson K, et al. The influence of sexual and social factors on the risk of Chlamydia trachomatis infections: a population-based serologic study. Sex Transm Dis. 1995; 22 (6), 355-63. doi: 10.1097/00007435-199511000-00007

[14] Holmes KK, Levine R, Weaver M. Effectiveness of condoms in preventing sexually transmitted infections. Bull World Health Organ. 2004; 82 (6), 454-61.

[15] Taylor SN, Liesenfeld O, Lillis RA, et al. Evaluation of the Roche cobas(R) CT/NG test for detection of Chlamydia trachomatis and Neisseria gonorrhoeae in male urine. Sex Transm Dis. 2012; 39, 543-9. doi: 10.1097/OLQ.0b013e31824e26ff

[16] Boyadzhyan B, Yashina T, Yatabe JH, et al. Comparison of the APTIMA CT and GC assays with the APTIMA combo 2 assay, the Abbott LCx assay, and direct fluorescent-antibody and culture assays for detection of Chlamydia trachomatis and Neisseria gonorrhoeae. J Clin Microbiol. 2004; 42, 3089-93. doi: 10.1128/ JCM.42.7.3089-3093.2004.

[17] Meyer T. Diagnostic Procedures to Detect Chlamydia trachomatis Infections. Microorganisms. 2016; 4 (3), 25. doi: 10.3390/microorganisms4030025

[18] Santos CG, Sabidó M, Leturiondo AL, et al. Development, validation and testing costs of an in-house real-time PCR assay for the detection of Chlamydia trachomatis. J Med Microbiol. 2017; 66 (3), 312-7. doi: 10.1099/jmm.0.000443

[19] Lourdes Mahilum-Tapay L, Vivian Laitila V, James J Wawrzyniak, et al. New point of care Chlamydia Rapid Test-bridging the gap between diagnosis and treatment: performance evaluation study. BMJ. 2017; 8, 335 (7631), 1190-4. doi: 10.1136/bmj.39402.463854.AE [20] Budai I. Chlamydia trachomatis: milestones in clinical and microbiological diagnostics in the last hundred years: a review. Acta Microbiol Immunol Hung. 2007; 54 (1), 5-22. doi: 10.1556/AMicr.54.2007.1.2

[21] Bébéar C, de Barbeyrac B. Genital Chlamydia trachomatis infections. Clin Microbiol Infect. 2009;15 (1), 4-10. doi: 10.1111/j.1469-0691.2008.02647.x

[22] Khan ER, Hossain MA, Paul SK, et al. Molecular diagnosis of genital Chlamydia trachomatis infection by polymerase chain reaction. Mymensingh Med J. 2011; $20(3), 362-5$. 